OPEN ACCESS

Edited by:

Alexis M. Kalergis,

Pontificia Universidad Católica de

chile, Chile

Reviewed by:

Vijayakumar Velu,

Emory University, United States

Ashutosh K. Mangalam,

The University of lowa, United States

*Correspondence:

Vainav Pate

patelv@nirrh.res.in

Jayanthi Shastri

jsshastri@gmail.com

Smita D. Mahale

smitamahale@hotmail.com

${ }^{\dagger}$ These authors share first authorship

¥These authors have contributed equally to this work

Specialty section:

This article was submitted to Viral Immunology,

a section of the journal

Frontiers in Immunology

Received: 21 October 2020

Accepted: 17 May 2021

Published: 14 June 2021

Citation:

Jagtap D, Bhor VM, Bhowmick S, Kasarpalkar N, Sagvekar P, Kulkarni B,

Pathak M, Chatterjee N, Dolas $P$, Palav H, Kaginkar S, Bhagat S,

Munshi I, Parikh S, Agrawal S,

Pawar C, Kaneria M, Mahale SD,

Shastri J and Patel V (2021)

sMAdCAM: IL-6 Ratio Influences

Disease Progression and Anti-Viral Responses in SARS-CoV-2 Infection.

Front. Immunol. 12:619906.

doi: 10.3389/fimmu.2021.619906

\section{sMAdCAM: IL-6 Ratio Influences Disease Progression and Anti-Viral Responses in SARS-CoV-2 Infection}

\author{
Dhanashree Jagtap ${ }^{1 \dagger}$, Vikrant M. Bhor ${ }^{2 \dagger}$, Shilpa Bhowmick ${ }^{3 \neq}$, Nandini Kasarpalkar ${ }^{2 f}$, \\ Pooja Sagvekar ${ }^{3}$, Bhalchandra Kulkarni ${ }^{1}$, Manish Pathak ${ }^{4}$, Nirjhar Chatterjee ${ }^{5}$, \\ Pranam Dolas ${ }^{6}$, Harsha Palav ${ }^{3}$, Snehal Kaginkar ${ }^{3}$, Sharad Bhagat ${ }^{3}$, Itti Munshi ${ }^{7}$, \\ Swapneil Parikh ${ }^{4}$, Sachee Agrawal ${ }^{5}$, Chandrakant Pawar ${ }^{6}$, Mala Kaneria ${ }^{5,6}$, \\ Smita D. Mahale ${ }^{1 *}$, Jayanthi Shastri ${ }^{4,5 *}$ and Vainav Patel ${ }^{3 *}$ \\ ${ }^{1}$ Department of Structural Biology Division, ICMR-NIRRH, Mumbai, India, ${ }^{2}$ Department of Molecular Immunology \& \\ Microbiology, Indian Council of Medical Research-National Institute for Research in Reproductive Health (ICMR-NIRRH), \\ Mumbai, India, ${ }^{3}$ Department of Biochemistry \& Virology, ICMR-NIRRH, Mumbai, India, ${ }^{4}$ Molecular Lab, Kasturba Hospital for \\ Infectious Diseases, Mumbai, India, ${ }^{5}$ Department of Microbiology, BYL Nair Hospital, Mumbai, India, ${ }^{6}$ Kasturba Hospital for \\ Infectious Diseases, Mumbai, India, ${ }^{7}$ Department of Primate Biology, ICMR-NIRRH, Mumbai, India
}

The role of sMAdCAM, an important gut immune migratory marker, remains unexplored in COVID-19 pathogenesis considering recent studies positing the gut as a sanctuary site for SARS-CoV-2 persistence. Thus, assimilating profiles of systemic inflammatory mediators with sMAdCAM levels may provide insights into the progression of COVID-19 disease. Also, the role of these markers in governing virus specific immunity following infection remains largely unexplored. A cohort $(n=84)$ of SARS-COV-2 infected individuals included a group of in-patients $(n=60)$ at various stages of disease progression together with convalescent individuals $(n=24)$ recruited between April and June 2020 from Mumbai, India. Follow-up of 35 in-patients at day 7 post diagnosis was carried out. Th1/Th2/Th17 cytokines along with soluble MAdCAM (sMAdCAM) levels in plasma were measured. Also, anti-viral humoral response as measured by rapid antibody test (IgG, $\lg M)$, Chemiluminescent Immunoassay (IgG), and antibodies binding to SARS-CoV-2 proteins were measured by Surface Plasmon Resonance (SPR) from plasma. IL-6 and sMAdCAM levels among in-patients inversely correlated with one another. When expressed as a novel integrated marker-sMIL index (sMAdCAM/IL-6 ratio)-these levels were incrementally and significantly higher in various disease states with convalescents exhibiting the highest values. Importantly, sMAdCAM levels as well as SMIL index (fold change) correlated with peak association response units of receptor binding domain and fold change in binding to spike respectively as measured by SPR. Our results highlight key systemic and gut homing parameters that need to be monitored and investigated further to optimally guide therapeutic and prophylactic interventions for COVID-19.

Keywords: COVID-19, inflammation, IL-6, sMAdCAM, sMIL index, antibodies, SPR 


\section{INTRODUCTION}

Having established itself in the human population, the SARSCoV-2 virus and its associated pathology (COVID19) is a subject of intense study with implications for public health globally (1-3). Inflammation, especially that mediated by 'cytokine storm' seems to be the primary etiological factor driving progression of COVID19 disease $(4,5)$. Persistent viral infection in sanctuaries such as the gut together with post-infection immunological sequelae is also emerging as relevant disease management issues that need to be addressed (6-8). Recent studies have identified key inflammatory cytokines such as IL- 6 and TNF- $\alpha$ that are dysregulated and appear to play a significant role in mediating pathology $(9,10)$. Also, the role, if any, of these cytokines and the associated inflammatory response in governing virus specific immunity remains largely unexplored following both symptomatic and asymptomatic infection. Understanding and identifying such signatures may guide current therapeutic and vaccine development efforts to ensure optimal disease management for this emerging pathogen. In this study, we undertook to examine cross-sectionally and longitudinally, profiles of inflammation, including a gut associated marker governing mucosal lymphocyte migration, across various stages of disease progression following SARS-CoV-2 infection.

Concurrent evaluation of virus specific humoral immunity was also carried out to understand the link between these profiles and generation, persistence and real-time binding kinetics of antibodies against various viral antigens. Our results highlight a putative immunological marker that seems to govern both disease progression and generation of anti-viral antibodies to the neutralizable receptor binding domain of SARS-CoV-2.

\section{MATERIALS AND METHODS}

\section{Study Population, Setting, and Data Collection}

A total of 60 in-patients and 24 convalescent individuals were recruited (from April to July, 2020), following informed consent, for the study from the isolation ward at Kasturba Hospital for Infectious Diseases (Municipal Corporation of Greater Mumbai). Pregnant women, prisoners, and children (less than 18 years of age) were excluded from the study. The Kasturba Hospital and ICMR-NIRRH institutional ethics committees approved this study. We obtained demographic data, clinical history at presentation, and laboratory results during admission.

Blood samples for the study were handled in accordance with ICMR guidelines for biosafety. Whole blood $(1-3 \mathrm{ml})$ was collected in EDTA vacutainers, and plasma was separated by centrifugation at $400 \mathrm{~g}$ for $10 \mathrm{~min}$. IgG and IgM antibodies against SARS-CoV-2 were detected in fresh plasma samples using Rapid test from either Voxpress (Voxtur Bio LTD, India) or Tell me fast (Biocan Diagnostics Inc., Canada) kits and also by ARCHITECT ${ }^{\mathrm{TM}}$ Abbott $^{\mathrm{TM}}$ (Abbott Diagnostics, USA) chemiluminescence immunoassay (CLIA) directed against SARS-CoV-2 anti-NC IgG. The remaining plasma samples were aliquoted and stored at $-80^{\circ} \mathrm{C}$ until batch analysis of cytokines, soluble MAdCAM, and antibody binding profile using Surface Plasmon Resonance.

\section{Cytokine Bead Array}

Human Th1/Th2/Th17 cytokines kit (BD CBA kit Cat no. 560484, NJ, USA) was used to measure IL-2, IL-4, IL-6, IL-10, TNF- $\alpha$, IFN- $\gamma$, and IL-17 cytokine levels in plasma of study participants.

Briefly, $50 \mu \mathrm{l}$ of plasma sample and standard were added to $50 \mu \mathrm{l}$ of capture bead mixture followed by addition of $50 \mu$ of human Th1/Th2/Th17 PE detection reagent. Following incubation at room temperature in dark for $3 \mathrm{~h}$, the assay tubes were washed with $1 \mathrm{ml}$ of wash buffer. The tubes were centrifuged at $200 \mathrm{~g}$ for $5 \mathrm{~min}$, and bead pellet was re-suspended in $300 \mu \mathrm{l}$ wash buffer and acquired using BD Accuri ${ }^{\mathrm{TM}}$ C6. Five thousand events were acquired gated on selected bead population, and analysis was carried out using BD C6 Accuri analysis software. GraphPad Prism was used to extrapolate individual cytokine concentrations in each sample.

\section{MAdCAM ELISA}

Human MAdCAM-1 DuoSet ELISA kit (R\&D Systems-DY605605. Minneapolis, USA) was used for estimation of soluble MAdCAM-1 in plasma in accordance with the manufacturer's protocol. Briefly, wells coated with capture antibody and reagent diluent (1\% BSA in PBS) were added for blocking. MAdCAM standard or 1:100 diluted plasma was added to wells and incubated overnight at $4^{\circ} \mathrm{C}$. Incubation with detection antibody was followed by addition of streptavidin-HRP which was allowed to react with a substrate solution to give chromogenic readout. Reaction was stopped using $2 \mathrm{~N} \mathrm{H} 2 \mathrm{SO} 4$, and absorbance was measured at 450 and $570 \mathrm{~nm}$ using Synergy $\mathrm{H} 1$ microplate reader (Biotek, Vermont, USA). Readings at $570 \mathrm{~nm}$ were subtracted from $450 \mathrm{~nm}$ and used to plot the standard curve. Plasma concentration of sMAdCAM was calculated by interpolating absorbance values and multiplying by dilution factor.

\section{Surface Plasmon Resonance Studies: Binding of COVID-19 Patients' Plasma to Immobilized SARS-CoV-2 Proteins-Spike, Receptor Binding Domain and Nucleocapsid}

The binding analysis of real time interaction between COVID-19 patients' plasma and SARS-CoV-2 proteins was carried out using the SPR spectrometer (Biacore 3000, GE Healthcare Bio-Sciences AB, Uppsala, Sweden). Flow cells of CM5 (carboxmethylated dextran matrix) sensor chip were first activated with $0.2 \mathrm{M}$ 1-ethyl-3-(3-dimethyl aminopropyl) carbodiimide hydrochloride and $0.05 \mathrm{M}$ N-hydroxysuccinimide. SARS-CoV-2 proteins (Spike, RBD, and Nucleocapsid) at a concentration of $20 \mu \mathrm{g} / \mathrm{ml}$ each in $10 \mathrm{mM}$ sodium acetate, $\mathrm{pH} 4.5$, were immobilized on the activated CM5 chip to about 1,400 response units (RUs), and the unreacted groups were blocked with $1.0 \mathrm{M}$ ethanolamine- $\mathrm{HCl}$, $\mathrm{pH}$ 8.5. Flow cell, which was not immobilized with any protein, served as negative control.

Human COVID-19 patients' plasma was divided into four groups based on their IgM/IgG status(Rapid Antibody Tests) and 
was used to determine the binding of antibodies to three SARSCoV-2proteins. Diluted plasma (1:10) in 10 mM HBS-EP, pH 7.4 running buffer was injected over Spike,RBD, and Nucleocapsid immobilized CM5 chip for $150 \mathrm{~s}$ (association phase) followed by subsequent dissociation for $150 \mathrm{~s}$ and recording of their spectra. All the experiments were performed at $25^{\circ} \mathrm{C}$, and the flow rate used was $20 \mu \mathrm{l} / \mathrm{min}$. The surface at the end of each experiment was regenerated using a $25 \mu \mathrm{l}$ pulse of $10 \mathrm{mM}$ Glycine-HCL, $\mathrm{pH}$ 2.5 , to remove any bound analyte. Sensorgrams were corrected by subtraction of the signal from the negative control surface as well as pre-COVID-19 plasma.

Data were evaluated using the BIA evaluation software program (4.1 version, GE Healthcare Bio-Sciences AB). Peak association response units (RUs) were recorded.

\section{Statistical Analysis}

Statistical analysis was performed in GraphPad Prism 8 using nonparametric tests. Statistical significance of differences between groups was assessed using Mann-Whitney U-test. Follow-up data from the same individual was compared with Wilcoxon matchedpaired signed rank test. Spearman's rank-order correlation was used to analyze the association between participant attributes and statistical significance was accepted at $\mathrm{p}<0.05$.

\section{RESULTS}

\section{Demographic and Clinical Characteristics of Study Participants}

During the period from April through July 2020, 60 in-patients with confirmed COVID-19 infection and under isolation were recruited at Kasturba Hospital. Of these 35 were followed up 7 days post admission. A total of 24 individuals, clinically documented to have been recovered and discharged, were also recruited as convalescents. Convalescent individuals had two consecutive negative respiratory swabs or were cleared of symptoms prior to discharge, and their blood samples were collected at least 2 weeks post discharge. The demographic, serological, and clinical characteristics of the patients are shown and summarized in Supplemental Table 1 and Table 1 respectively.
As apparent from these tables our study cohort included individuals that were analyzed in a cross-sectional, longitudinal (0 day and 7 day time points) manner and at clinically distinct (in-patients and convalescent) stages of disease progression. Also, as expected, males pre-dominated, and individuals with co-morbidities constituted a significant proportion $(28 / 66 ; 42 \%)$ of the cohort. Serological data evaluated through both a rapid test (IgG, IgM) and CLIA (anti-NC IgG) showed an overall concordance of $77 \%$ for IgG and also allowed us to group these individuals based on IgG and/or

$\operatorname{IgM}$ positivity. When grouped in this fashion it was interesting to note that while a majority of convalescent individuals $\sim 88 \%$ (21 out of 24 ) were seropositive for SARSCoV-2 about $12 \%$ (three out of 24) were seronegative both by CLIA and rapid test (Table 1 and Supplemental Table 1). Also, CLIA data for circulating anti-viral antibodies trended lower in convalescent individuals but wasn't statistically significant.

\section{Inflammatory Cytokine Profile and Gut Migration}

As shown in Figures 1A, C and Supplemental Figures 1A, B, elevated levels of IL- 6 and TNF- $\alpha$ were observed both in in-patients and, of note, in convalescent individuals compared to healthy individuals. Also, these levels spanned a range of seropositivity in terms of virus specific IgG and/or IgM. Also, we noticed a significant reduction of IL-6, but not TNF- $\alpha$, as seropositivity transitioned from double negative to IgG, IgM double positive, and finally to class switched (IgG single positive) responses in convalescent individuals. Increased levels of these cytokines did not correlate with initial Ct values, each other, and that of any other cytokine measured (Supplemental Figures 2, 3). When these levels were examined longitudinally (Figures 1C, E and Supplemental Figure 1C) in a group of 11 individuals that were followed at day 0 (day of diagnosis) and day 7 (post diagnosis), we noticed a declining trend for IL- 6 but not TNF- $\alpha$.

Additionally, and for the first time, we report on gut migratory marker sMAdCAM (Figures 1B, D), which appeared to be circulating at levels lower than those seen in healthy individuals and convalescents. Also there was no difference between sMAdCAM levels in 34 individuals that were followed at day 0

TABLE 1 | Abridged clinical data of study participants.

\begin{tabular}{|c|c|c|c|}
\hline & In-patients $(n=60)$ & Convalescent (Group V) ( $=24)$ & Uninfected Healthy \\
\hline Number of Participants & 60 (35 follow-up $\left.{ }^{b}\right)$ & 24 & 19 \\
\hline Age $^{a}$ (years) & $46(18-74)$ & $34(20-55)$ & $30(24-47)$ \\
\hline \multirow[t]{2}{*}{ Gender } & 15 Women (25\%) & 5 Women (21\%) & 9 Women (47\%) \\
\hline & 45 Men (75\%) & 19 Men (79\%) & 10 Men (53\%) \\
\hline Days post PCR test until sampling ${ }^{a}$ & $0-19(6)$ & 19-62 (37) & $\mathrm{N}$ \\
\hline CLIA index ${ }^{a}$ & $1.55-9.69(5.93)$ & $2.58-9.47(4.48)$ & $N$ \\
\hline \multicolumn{4}{|l|}{ Grouping } \\
\hline IlgM-lgG- & 38 & 3 & 19 \\
\hline IIIgM+lgG- & 13 & - & - \\
\hline III lgM+lgG+ & 25 & 2 & - \\
\hline IV IgM-lgG+ & 17 & 19 & - \\
\hline
\end{tabular}

${ }^{a}$ Data are expressed as the median (range). ${ }^{b}$ Blood sampling of 35 study participants was carried out on day 0 and day 7 and considered separately (as follow-up samples) for analysis. Sero-grouping data was not available for one in-patient (follow-up). N, Not applicable. 


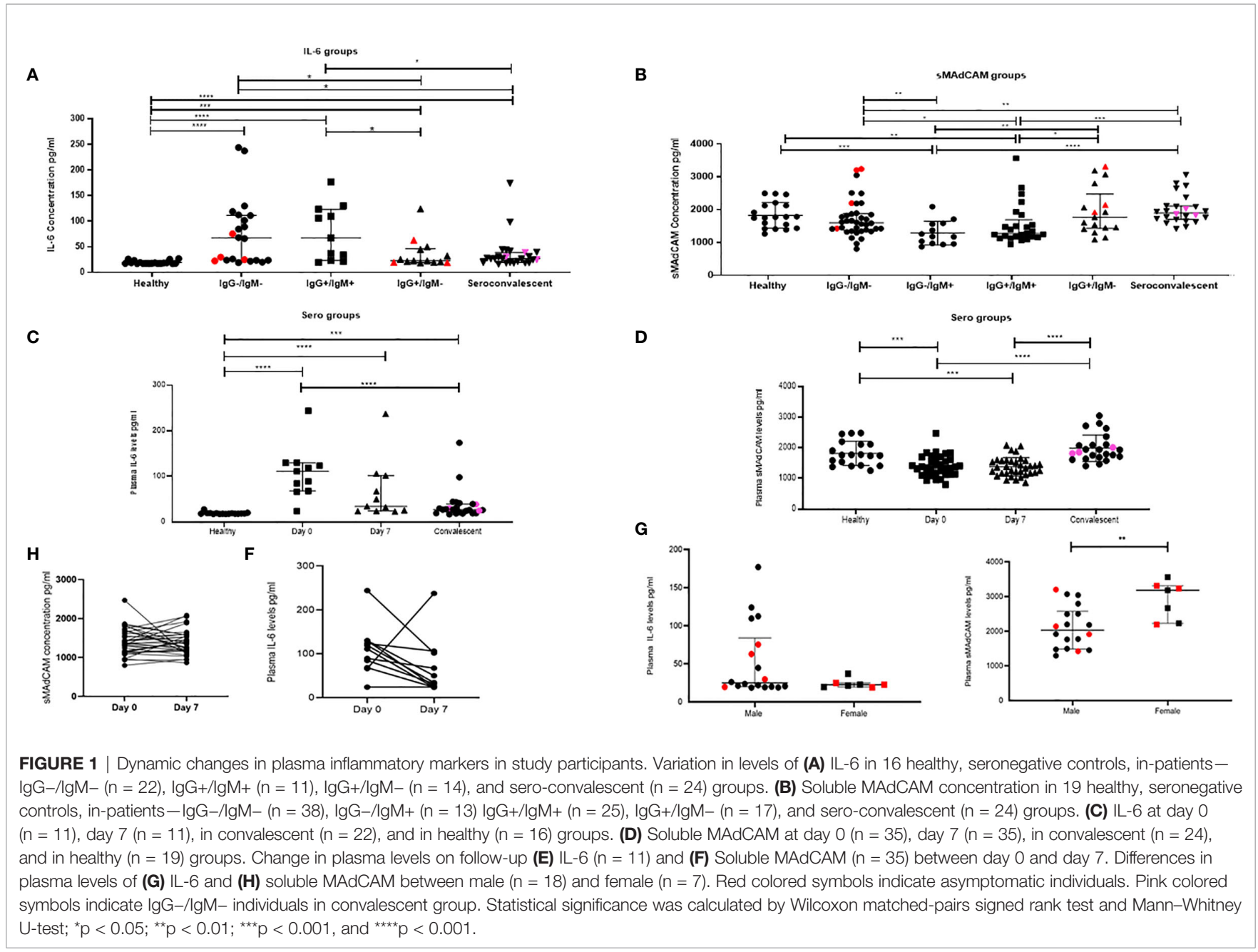

and day 7 (Figures 1D, F). Interestingly however (Figure 1B), in contrast and conversely with respect to IL-6, sMAdCAM levels seemed to reduce (compared to healthy controls) following early infection (IgM single positive and IgG, IgM double positive stages) and attain restoration in class switched individuals (IgG single positive and convalescents). Further, LPS levels, evaluated in a subset of in-patients and convalescents, did not vary signifying absence of microbial translocation associated gut inflammation (Supplemental Figure 4).

Next, analyzing these individuals based on disease progression clearly demonstrated a declining circulating IL-6 but not TNF- $\alpha$ concentration (Figure 1 and Supplemental Figure 1). Stratification of the in-patient data (Figures 1G, H) on the basis of gender revealed a marked difference in circulating levels of IL- 6 and sMAdCAM where women had significantly lower and higher levels of these respectively.

\section{sMAdCAM: IL-6 Ratio (sMIL Index): A Novel Biomarker for Disease Progression}

Results obtained from the heretofore described analyses (Figure 1) suggested that sMAdCAM and IL-6 levels independently seemed to track (Figure 2) with disease progression, albeit in opposing directions. Suspecting an underlying association between both of these apparently unrelated signatures, we examined the utility of their being combined as an integrated marker that could better correlate and discriminate between various stages of infection.

Remarkably, when levels of both these analytes were expressed as a sMIL ratio (sMAdCAM/IL-6), we observed (Figure 3) a clear delineation of in-patient and convalescent data into discrete categories based on disease progression (Figures 3A, B), gender (Figure 3C), time from infection (Figure 3D), and intriguingly serological status (Figure 3E). These observations seemed to posit a role for this index in the development of antibodies against SARS-CoV-2.

\section{Anti-Viral Responses and the Role of Inflammatory Markers}

Anti-SARS-CoV-2 responses were measured using surface plasmon resonance (SPR) that allowed us to obtain a sensitive and real-time evaluation of antibody binding to viral Spike, RBD, and NC proteins. First, we demonstrated that SPR was indeed picking up antibody binding through the observed concordance between CLIA profile and peak association (response unit i.e. $\mathrm{RU}$ ) indicating viral protein binding observed in our prospective 
A

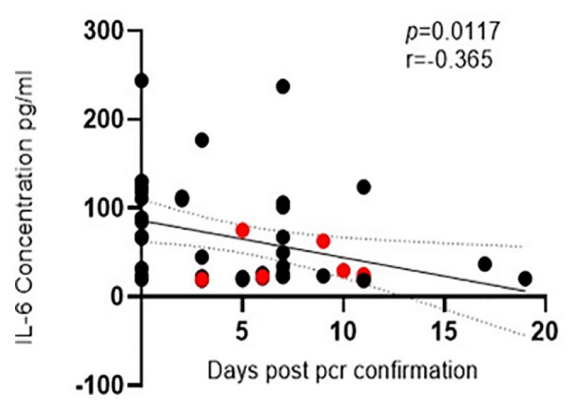

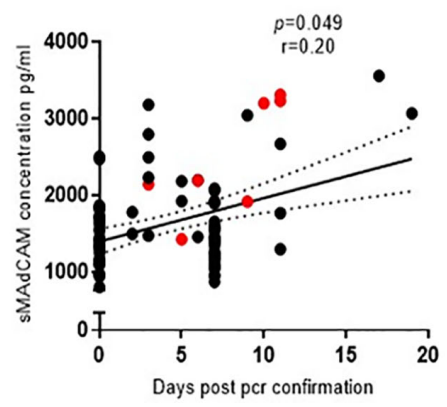

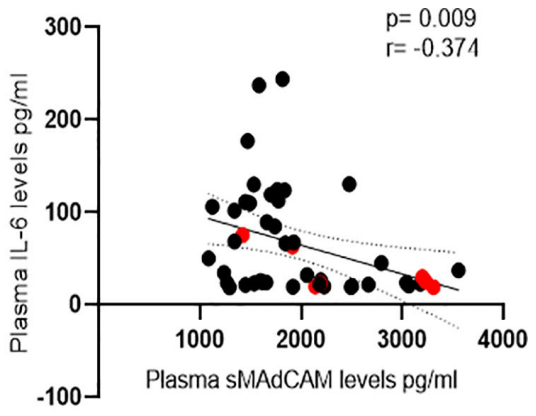

FIGURE 2 | Association of IL-6 and soluble MAdCAM in SARS-CoV-2 infection. (A, B) Association of (A) IL-6 levels ( $n=47)$ and (B) soluble MAdCAM levels ( $n=95)$ in plasma of SARS-CoV-2 infected study participants with days since SARS-CoV-2 confirmation by PCR. (C) Association of IL-6 and soluble MAdCAM among in-patients' $(n=47) r$ indicates strength of correlation; $p$ indicates significance. Negative $r$ value indicates inverse correlation. Red colored symbols represent asymptomatic individuals. Statistical significance was calculated by Spearman correlation analysis.

A

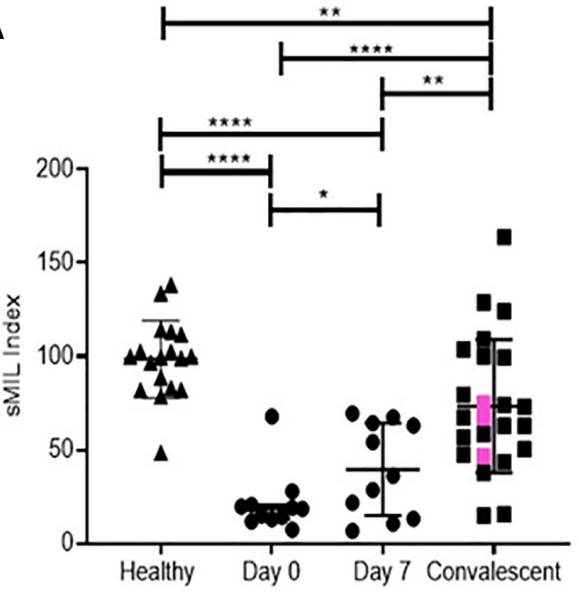

D

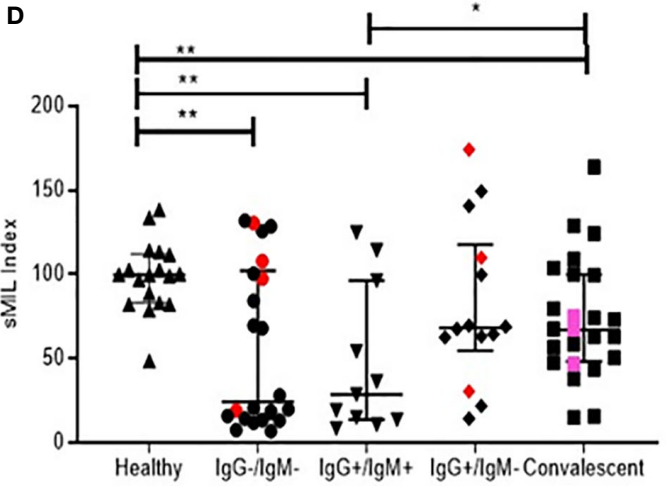

B

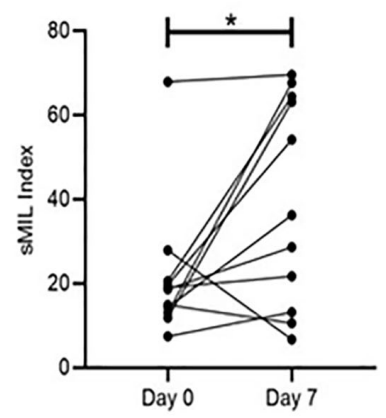

C

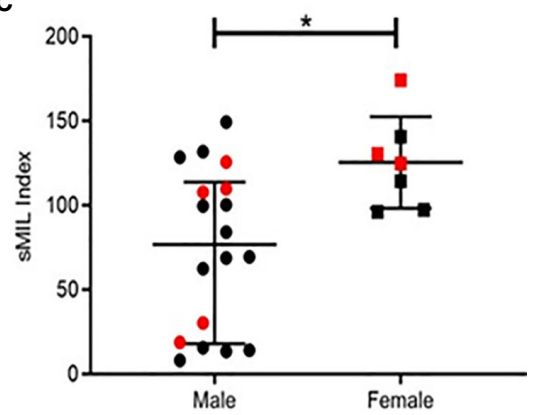

$\mathbf{E}$

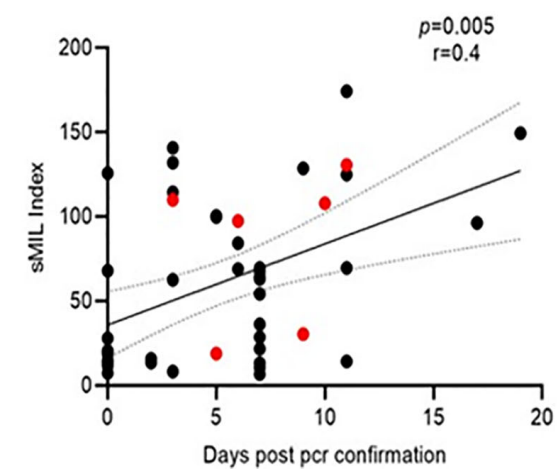

FIGURE 3 | Distribution of peak RU across various groups of study participants. Also the resolution of figures are satisfactory (A) Variation in sMIL index at day 0 $(n=11)$, day $7(n=11)$, in convalescent $(n=22)$, and in healthy $(n=16)$ groups. Pink colored symbols indicate lgG-/gM- individuals in convalescent group. (B) Change in SMIL index between day $0(n=11)$ and day $7(n=11)$ expressed per individual.(C) Differences in sMIL index between male $(n=18)$ and female $(n=7)$. (D) Comparison of sMIL index between healthy $(n=16)$, lgG-/lgM- $(n=22), \lg G+/ \lg M+(n=11), \lg +/ \operatorname{lgM}-(n=14)$, and convalescent $(n=22)$ groups. (E) Association of SMIL index with days since SARS-CoV-2 confirmation by PCR $(n=47)$. Red colored symbols represent asymptomatic individuals. Statistical analysis was performed using Graphpad Prism 8.0. Wilcoxon matched-pairs signed rank test was used to compare paired samples of day 0 and day 7 . MannWhitney U-test was used to compare unpaired groups. ${ }^{*} \mathrm{p}<0.05,{ }^{* \star} \mathrm{p}<0.01$ and ${ }^{\star \star \star *} \mathrm{p}<0.001$. Correlation analysis was performed using non-parametric Spearman rank correlation test. 
cohort (Figures 4A-D). Further, we observed that individuals that were below the level of detection in the CLIA assay (Y-axis value 0) did have detectable binding using SPR.

We then proceeded to stratify plasma binding data (peak association RU) based on IgM/IgG serostatus (Figures $4 \mathbf{E}-\mathbf{G}$ ) as well as on disease progression (Figures $\mathbf{4 H}-\mathbf{J}$ ). In the case of the later, we observed significant increases in binding by day 7 for all 3 viral targets with increased responses against NC and RBD compared to $S$ followed by steep declines of these responses in convalescent plasma.

When grouped cross-sectionally by serostatus we observed (Figures 4E-G), as would be expected, an increase in binding from the IgG-IgM- stage to the single positive, post classswitched stage (IgM-IgG+). Also, as observed in the smaller prospective cohort, peak association RUs were higher for NC and RBD. For all three viral proteins, these peak association RUs were significantly lower in convalescent individuals with presumably mature memory B cells and viral clearance.

Having already demonstrated the importance of circulating IL-6 and sMAdCAM either in combination (sMIL index, Figure 3) or in isolation (Figures 1, 2) in influencing disease progression and antiviral antibody production, we investigated the possibility of identifying a specific viral target (Spike, RBD, or NC) whose binding response was associated and thus potentially attributable to these markers. Indeed, we were able to identify (Figure 5) a strong correlation between sMAdCAM levels and peak association rate of responses against the viral neutralization target, $\mathrm{RBD}$ in convalescent individuals. Also, we observed a relatively weaker but suggestive association with fold changes (day 0 and day 7 ) in sMIL index with that of viral Spike protein binding in plasma of the follow-up cohort.

\section{DISCUSSION}

Our study is the first to identify a putative integrated marker of inflammation and homeostaticimmune migration that associates both with COVID-19 disease progression and generation of potentially neutralizing antibody (11) responses against SARS-CoV-2.

Considering the already demonstrated role of inflammatory cytokines in the development of pathology of COVID-19 (12), we undertook to evaluate how inflammatory profiles (Th1/Th2/ Th17) correlated both cross-sectionally and longitudinally with varying disease states. Further, the role of an important emerging mediator of gut inflammation and mucosal homing, sMAdCAM, was examined in light of its significant role in the pathology of chronic inflammatory conditions including those with viral

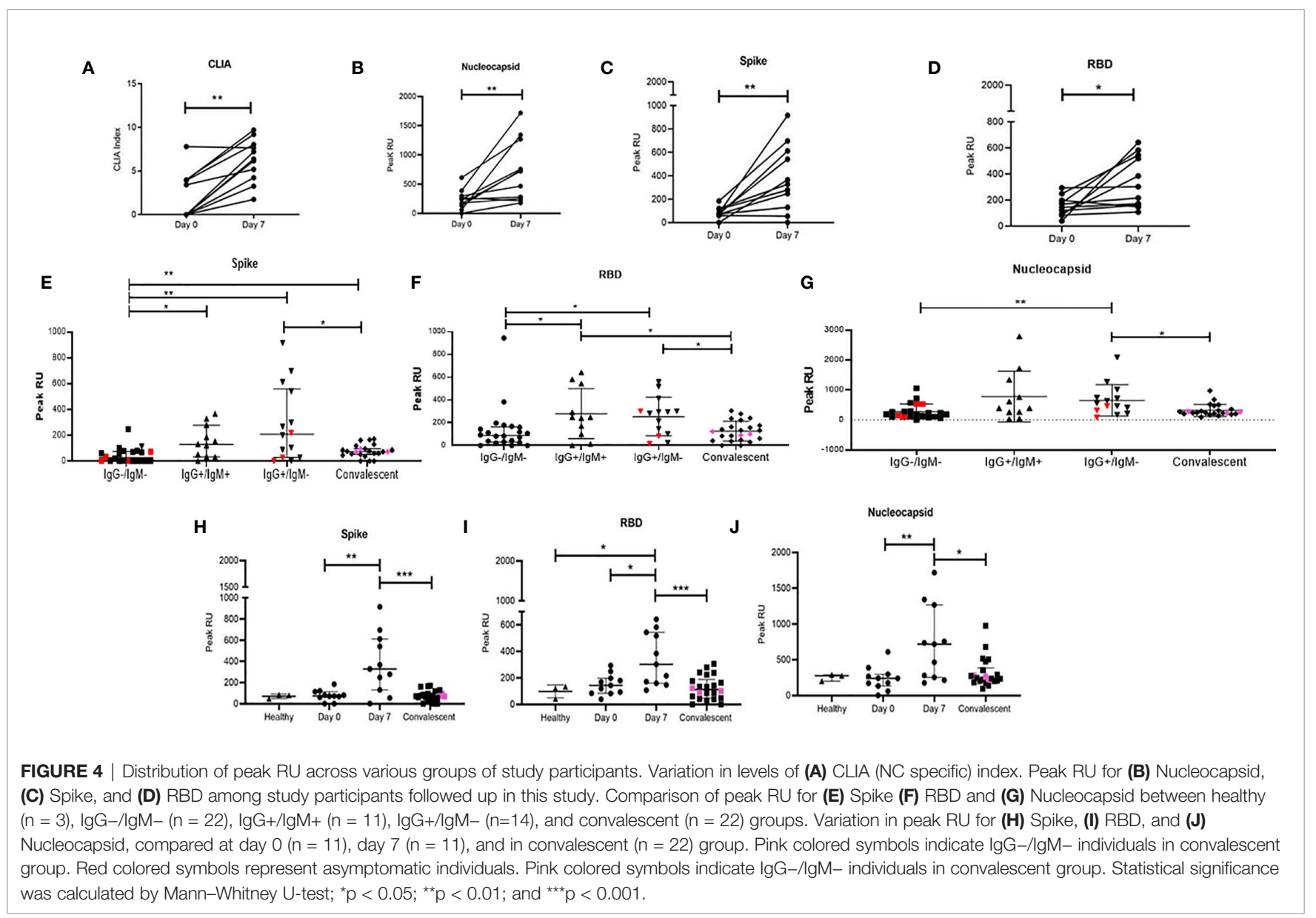


A

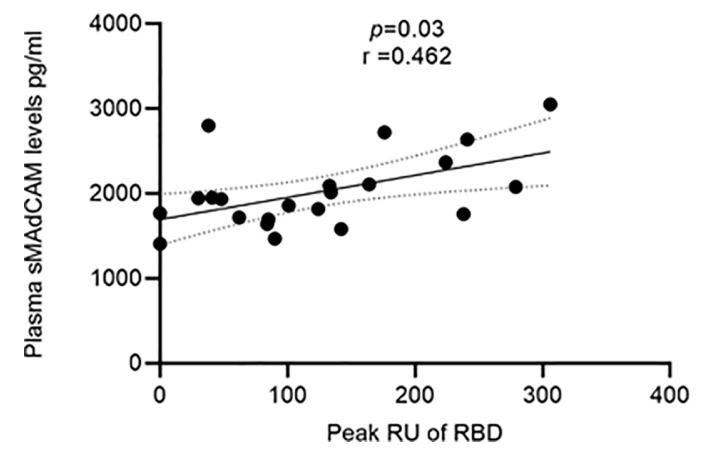

B

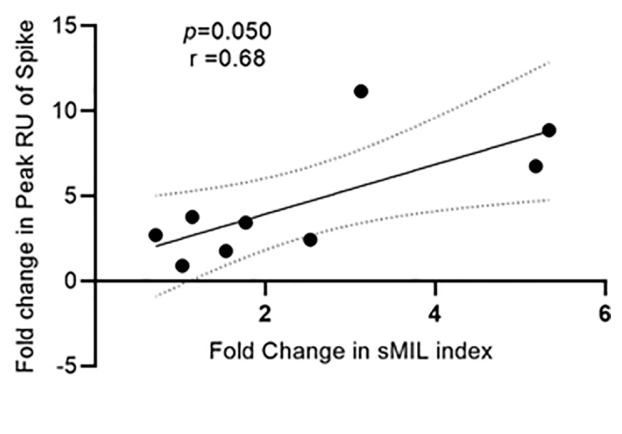

FIGURE 5 | Association of SMAdCAM levels/sMIL index and virus specific binding in plasma. (A) Association of plasma MAdCAM levels with peak RU (association rate) of RBD binding among convalescent study participants. (B) Association of fold change between peak RU for spike and sMIL index among study participants ( $n=9$ out of 11 ) who were followed up. Fold change was calculated by dividing day 7 values with those of day 0 . Fold change in peak RU of two individuals could not be calculated as day 0 values were undetectable (zero). Correlation analysis performed using non-parametric Spearman rank correlation test. $r$ indicates strength of correlation; $p$ indicates significance.

etiology (13-17). This aspect of our study is especially noteworthy in the context of accumulating evidence for the gut as a potential sanctuary site for SARS-CoV-2 and persistent immunological sequelae observed in apparently virus free individuals (18-20). Whether altered sMAdCAM levels reflect changes in migration to various mucosal sites (gut to respiratory mucosa and vice versa) in response to SARS-CoV-2 viral infection remains to be established. Additionally, its role in microbial translocation, a well-established pathology for viruses such as $\operatorname{HIV}(21,22)$ and recently described in the context of SARS-CoV-2 infection needs to be evaluated (9).

Our in-patient cohort included participants at various stages of disease progression and included a group of 11 individuals where inflammatory profiles as well as anti-viral responses could be measured in a prospective manner relatively early after infection. These profiles were compared to a group of convalescent, disease free individuals that were presumed to have recovered from infection. We were also able to group study participants on the basis of anti-viral IgG and IgM responses which enabled a concurrent evaluation of inflammatory signatures with the development of immune responses.

Our cytokine data highlights and confirms the predominance of IL-6 as an inflammatory mediator of pathology early following infection evinced through declining levels that trended inversely both with disease progression and transitioning of double negative (IgM-IgG) to IgG single positive anti-viral responses. Together with TNF- $\alpha$, it was interesting to note that similar dysregulated levels of these cytokines were observed in apparently 'convalescent' individuals that, in some cases, had been discharged following not just resolution of symptoms but also repeated negative RT-PCR diagnoses in respiratory swabs. These observations may prove useful in optimizing screening procedures of donors for plasma therapy that are often drawn from a pool of convalescent individuals (23), that as of now, are simply screened for the ability to mount anti-viral antibodies without accounting for sequelae such as ongoing inflammatory cytokine dysregulation. Also, stark differences in circulating IL-6, but not TNF- $\alpha$ levels, were observed in male participants who exhibited higher levels of this cytokine. This observation is consistent with reports of milder pathology and faster recuperation following infection in women (24-26). Further, the small size of our female cohort with heterogenous age (implying both pre and post-menopausal status) limited our ability to delineate a role for sex hormones in the relatively lower inflammatory signature observed in women enrolled in our study. However, it is interesting to note that a very tight grouping for IL-6 levels was observed in these women, irrespective of their age (range, 20-72 years; Median, 38 years).

Our results with sMAdCAM are interesting in light of the limited data available from select gut associated inflammatory pathologies (27-30); however, when stratified by disease state (inpatient or convalescent status) as well as by gender an intriguing trend for this marker was observed. Both convalescent individuals and females had significantly elevated levels of sMAdCAM and conversely lower levels of IL-6. This observation led us to investigate whether the relationship between these two markers reflected an inflammatory/homeostatic dichotomy where favorable disease progression or convalescence was characterized by relative levels (or ratios) of these mediators. Indeed, our analysis, heretofore unreported, confirmed that only the ratio (but not levels in isolation) of sMAdCAM/IL-6 (sMIL index) was able to stratify infected individuals into varying disease states cross-sectionally, longitudinally, by gender, and most importantly by immune competence as measured by class switched IgG anti-viral responses. Whether the sMIL index would serve as a similar correlate in other diseases involving inflammation and mucosal homeostasis remains to be evaluated.

The apparent link between sMIL index and class switched antibodies detected in advanced disease progression and convalescent individuals drove us to characterize plasma borne anti-viral humoral responses in our cohort against multiple viral targets NC, Spike, and RBD, that are significant for diagnosis and 
vaccine development (31). Real-time binding profiles, through surface plasmon resonance (SPR), offered a more realistic recapitulation of in-vivo binding and were thus generated directly by flowing plasma over immobilized viral proteins. This analysis first revealed a good concordance between detected peak association RU of anti-NC responses and CLIA IgG indices obtained for the same target. Of note however, is the observation that the SPR assay was able to detect binding against NC even in individuals that had undetectable levels as measured by CLIA, highlighting its possible utility as an early sero-diagnostic tool $(32,33)$.

When comparing peak association RUs across disease progression (longitudinally) or disease states (in-patients and convalescents), responses to all three targets were clearly detectable by day 7 following diagnosis of infection. Also, convalescents uniformly had lower levels of these antibodies in circulation in keeping with their presumed viral clearance (34). Overall, in concordance with previous reports for both SARS and SARS-CoV-2 using endpoint assays $(35,36)$, levels of anti-NC binding were detected early following infection at high levels and appeared to be more persistent than those of anti-Spike responses. When stratified into groups reflecting maturation status of antibody responses (IgM/IgG status) we noticed gratifyingly that the relatively lower and less persistent anti-Spike responses could be explained by the observation that both anti-NC and RBD binding responses, but not anti-Spike responses, were significantly observed at the IgM+IgG+ (double positive) stage suggesting earlier and probably better memory cell generation for these viral targets. We propose further exploration of these mechanisms may also help explain the observed rapid loss or waning to undetectable levels of these antibodies in some convalescent individuals $(37,38)$ and also reported for SARS infection (39).

Finally, the observed correlation between sMAdCAM and SMIL index with peak anti-RBD RUs and fold change in anti-Spike responses respectively further confirm the association of apparently disparate arms of the immune response in generating anti-viral immunity and highlight the importance of inflammatory/ mucosal homeostatic markers as putative positive immune correlates for efficacious vaccines (40-43).

\section{LIMITATIONS}

The limitations of this study include a modest sized cohort that may reduce the impact of the observations and require validation through larger prospective studies. Also, the follow-up data was based on 35 of 60 in-patients due to limited availability of sequential samples. Further, the date of diagnosis was considered as the onset of disease progression which may not have been the case in all cases.

\section{CONCLUSION}

Our study is the first to report on the role for sMAdCAM in the pathology of and immunity against SARS-CoV-2 as well as on its utility together with IL-6 levels (sMIL index) in serving as an integrative disease progression marker for COVID-19.

\section{DATA AVAILABILITY STATEMENT}

The raw data supporting the conclusions of this article will be made available by the authors without undue reservation subject to local ethics approval if necessary.

\section{ETHICS STATEMENT}

The studies involving human participants were reviewed and approved by the Institution Ethics Committees of both ICMRNIRRH and Kasturba Hospital. The patients/participants provided their written informed consent to participate in this study.

\section{AUTHOR CONTRIBUTIONS}

ShiB and NK contributed to sample processing, assay running, data analysis. DJ and VB helped conceive the study, recruited participants, performed assays, analyzed data and helped write the paper. PS performed participant recruitment and data analysis. BK performed SPR and data analysis. HP, SK, ShaB, and IM processed samples. MK, MP, NC, SA, CP, and PD assisted with participant recruitment and sample co-ordination. SP assisted with writing the manuscript. JS and SM helped conceive and co-ordinate the study. VP helped conceive the study, recruited participants, performed assays, analyzed data and figure generation, compiled results and wrote the manuscript. All authors contributed to the article and approved the submitted version.

\section{ACKNOWLEDGMENTS}

We are extremely grateful to Director General, Indian Council of Medical Research (ICMR), Ministry for Health \& Family Welfare, Government of India for encouraging and supporting with resources, the pursuit of important research questions relevant to COVID-19 pathogenesis. Also, none of this work would have been possible without the active participation and support of participants to whom we are grateful.

\section{SUPPLEMENTARY MATERIAL}

The Supplementary Material for this article can be found online at: https://www.frontiersin.org/articles/10.3389/fimmu.2021.619906/ full\#supplementary-material 


\section{REFERENCES}

1. WHO. Coronavirus Disease (COVID-19) Situation Report - 209. (2020). Available at https://www.who.int/emergencies/diseases/novel-coronavirus2019/situation-reports

2. Nuzzo J, Moss B, Kahn J, Rutkow L. John Hopkins Coronavirus Research Center. (2020). Available at https://coronavirus.jhu.edu/

3. Gupta N, Praharaj I, Bhatnagar T, Thanga JWV, Chauhan H, Kulkarni S, et al. Severe Acute Respiratory Illness Surveillance for C 2019, India, 2020. Indian J Med Res (2020) 151:236-40. doi: 10.4103/ijmr.IJMR_1035_20

4. Han H, Ma Q, Li C, Liu R, Zhao L, Wang W, et al. Profiling Serum Cytokines in COVID-19 Patients Reveals IL-6 and IL-10 Are Disease Severity Predictors. Emerg Microbes Infect (2020) 9:1123-30. doi: 10.1080/22221751.2020.1770129

5. Ragab D, Salah Eldin H, Taeimah M, Khattab R, Salem R. The COVID-19 Cytokine Storm; What We Know So Far. Front Immunol (2020) 11:1446. doi: 10.3389/fimmu.2020.01446

6. Wu Y, Guo C, Tang L, Hong Z, Zhou J, Dong X, et al. Prolonged Presence of SARS-CoV-2 Viral RNA in Faecal Samples. Lancet Gastroenterol Hepatol (2020) 5:434-5. doi: 10.1016/S2468-1253(20)30083-2

7. Effenberger M, Grabherr F, Mayr L, Schwaerzler J, Nairz M, Seifert M, et al. Faecal Calprotectin Indicates Intestinal Inflammation in COVID-19. Gut (2020) 69:1543-4. doi: 10.1136/gutjnl-2020-321388

8. Uzzan M, Soudan D, Peoc'h K, Weiss E, Corcos O, Treton X. Patients With COVID-19 Present With Low Plasma Citrulline Concentrations That Associate With Systemic Inflammation and Gastrointestinal Symptoms. Dig Liver Dis (2020) 52(10):1104-5. doi: 10.1016/j.dld.2020.06.042

9. Arunachalam PS, Wimmers F, Mok CKP, Perera RAPM, Scott M, Hagan T, et al. Systems Biological Assessment of Immunity to Mild Versus Severe COVID-19 Infection in Humans. Science (2020) 6261:1-18. doi: 10.1126/science.abc6261

10. Hadjadj J, Yatim N, Barnabei L, Corneau A, Boussier J. Impaired Type I Interferon Activity and Inflammatory Responses in Severe COVID-19 Patients. Science (80- ) (2020) 724:718-24. doi: 10.1126/science.abc6027

11. Salazar E, Kapur V, Musser JM. Convalescent Plasma anti-SARS-CoV-2 Spike Protein Ectodomain and Receptor Binding Domain IgG Correlate With Virus Neutralization. J Clin Invest (2020) 130(12):6728-38. doi: 10.1172/JCI141206

12. García LF. Immune Response, Inflammation, and the Clinical Spectrum of COVID-19. Front Immunol (2020) 11:1441. doi: 10.3389/fimmu.2020.01441

13. Graham J, Trovato F, Erminelli D, Huang X, Liberal R, Vergani D, et al. THU037-Soluble MadCAM-1 as a Novel Marker of Hepatic Endothelial Dysfunction in Acutely Decompensated Cirrhosis. J Hepatol (2019) 70:e176. doi: 10.1016/s0618-8278(19)30320-2

14. Paul S, Williet N, Di Bernado T, Berger AE, Boschetti G, Filippi J, et al. Soluble Mucosal Addressin Cell Adhesion Molecule 1 and Retinoic Acid Are Potential Tools for Therapeutic Drug Monitoring in Patients With Inflammatory Bowel Disease Treated With Vedolizumab: A Proof of Concept Study. J Crohn's Colitis (2018) 12:1089-96. doi: 10.1093/ecco-jcc/jjy077

15. Nawaz F, Livia RG, Jocelyn CR, Ronke O, Alia S, Aftab AA, et al. MadCAM Costimulation Through Integrin- $\alpha 4 \beta 7$ Promotes HIV Replication. Mucosal Immunol (2018) 11:1342-51. doi: 10.1038/s41385-018-0044-1.Senolytics

16. Kasarpalkar N, Deb B, Kumar P, Bhor VM. The Role of Integrin $\alpha 4 \beta 7$ Signaling in Human Immunodeficiency Virus-1 Pathogenesis and Viral Entry in Primary Cd4+ T Cells As Revealed by Comparative Phosphoproteomic Signatures. OMICS (2020) 24:437-50. doi: 10.1089/omi.2019.0196

17. Kasarpalkar NJ, Bhowmick S, Patel V, Savardekar L, Agrawal S, Shastri J, et al. Frequency of Effector Memory Cells Expressing Integrin $\alpha 4 \beta 7$ Is Associated With Tgf- $\beta 1$ Levels in Therapy Naïve HIV Infected Women With Low Cd4 + T Cell Count. Front Immunol (2021) 12:651122. doi: 10.3389/fimmu.2021.651122

18. Trottein F, Sokol H. Potential Causes and Consequences of Gastrointestinal Disorders During a SARS-CoV-2 Infection. Cell Rep (2020) 32(3):107915. doi: 10.1016/j.celrep.2020.107915

19. Greenhalgh T, Knight M, A'Court C, Buxton M, Husain L. Management of PostAcute covid-19 in Primary Care. BMJ (2020) 370:m3026. doi: 10.1136/bmj.m3026

20. Ramlall V, Thangaraj PM, Meydan C, Foox J, Butler D, Kim J, et al. Immune Complement and Coagulation Dysfunction in Adverse Outcomes of SARS-CoV-2 Infection. Nat Med (2020) 26(10):1609-15. doi: 10.1038/s41591-020-1021-2

21. Brenchley JM, Price DA, Schacker TW, Asher TE, Silvestri G, Rao S, et al. Microbial Translocation Is a Cause of Systemic Immune Activation in Chronic HIV Infection. Nat Med (2006) 12:1365-71. doi: 10.1038/nm1511
22. Salwe S, Padwal V, Nagar V, Patil P, Patel V. T Cell Functionality in HIV-1, HIV-2 and Dually Infected Individuals: Correlates of Disease Progression and Immune Restoration. Clin Exp Immunol (2019) 198(2):233-50. doi: 10.1111/ cei. 13342

23. Mukherjee A, Agarwal A. Study to Assess the Efficacy and Safety of Convalescent Plasma in Moderate COVID-19 Disease. Clin Trials Regist India (2020), 1-4.

24. Jin JM, Bai P, He W, Wu F, Liu XF, Han DM, et al. Gender Differences in Patients With COVID-19: Focus on Severity and Mortality. Front Public Heal (2020) 8:152. doi: 10.3389/fpubh.2020.00152

25. Conti P, Younes A. Coronavirus cov-19/sars-cov-2 Affects Women Less Than Men: Clinical Response to Viral Infection. J Biol Regul Homeost Agents (2020) 34:339-43. doi: 10.23812/Editorial-Conti-3

26. Takahashi T, Ellingson MK, Wong P, Israelow B, Lucas C, Klein J, et al. Sex Differences in Immune Responses to SARS-CoV-2 That Underlie Disease Outcomes. (2020) 588(7837):315-20. doi: 10.1038/s41586-020-2700-3

27. Battat R, Dulai PS, Vande Casteele N, Evans E, Hester KD, Webster E, et al. Biomarkers Are Associated With Clinical and Endoscopic Outcomes With Vedolizumab Treatment in Ulcerative Colitis. Inflamm Bowel Dis (2019) 25:410-20. doi: 10.1093/ibd/izy307

28. Hassan-Zahraee M, Banerjee A, Cheng JB, Zhang W, Ahmad A, Page K, et al. Anti-MAdCAM Antibody Increases $\beta 7+$ T Cells and CCR9 Gene Expression in the Peripheral Blood of Patients With Crohn's Disease. J Crohn's Colitis (2018) 12:77-86. doi: 10.1093/ecco-jcc/jjx121

29. Sandborn WJ, Lee SD, Tarabar D, Louis E, Klopocka M, Klaus J, et al. Phase II Evaluation of anti-MAdCAM Antibody PF-00547659 in the Treatment of Crohn's Disease: Report of the OPERA Study. Gut (2018) 67:1824-35. doi: 10.1136/gutjnl-2016-313457

30. Nishida Y, Hosomi S, Yukawa T, Otani K, Nagami Y, Tanaka F, et al. Serum Interleukin-6 Level Is Associated With Response to Infliximab in Ulcerative Colitis. Scand J Gastroenterol (2018) 53(5):579-85. doi: 10.1080/00365521. 2017.1403647

31. Wu F, Liu M, Wang A, Lu L, Wang Q, Gu C, et al. Evaluating the Association of Clinical Characteristics With Neutralizing Antibody Levels in Patients Who Have Recovered From Mild COVID-19 in Shanghai, China. JAMA Intern Med (2020) 180(10):1356-62. doi: 10.1001/jamainternmed.2020.4616

32. Djaileb A, Charron B, Jodaylami MH, Thibault V, Coutu J, Stevenson K, et al. A Rapid and Quantitative Serum Test for SARS-CoV-2 Antibodies With Portable Surface Plasmon Resonance Sensing. ChemRxiv (2020). doi: $10.26434 /$ chemrxiv.12118914

33. Moberg A, Lager A, Hämäläinen MD, Jarhede T. Increased Sensitivity of SPR Assays in Plasma Through Efficient Parallel Assay Optimization. J Pharm BioMed Anal (2013) 78-79:224-32. doi: 10.1016/j.jpba.2013.02.018

34. Hou H, Wang T, Zhang B, Luo Y, Mao L, Wang F, et al. Detection of IgM and IgG Antibodies in Patients With Coronavirus Disease 2019. Clin Transl Immunol (2020) 9:1-8. doi: 10.1002/cti2.1136

35. Leung DTM, Tam FCH, Chun HM, Chan PKS, Cheung JLK, Niu H, et al. Antibody Response of Patients With Severe Acute Respiratory Syndrome (SARS) Targets the Viral Nucleocapsid. J Infect Dis (2004) 190:379-86. doi: 10.1086/422040

36. Burbelo PD, Riedo FX, Morishima C, Rawlings S, Smith D, Das S, et al. Sensitivity in Detection of Antibodies to Nucleocapsid and Spike Proteins of Severe Acute Respiratory Syndrome Coronavirus 2 in Patients With Coronavirus Disease 2019. J Infect Dis (2020) 222:206-13. doi: 10.1093/infdis/jiaa273

37. Ibarrondo FJ, Fulcher JA, Goodman-Meza D, Elliott J, Hofmann C, Hausner MA, et al. Rapid Decay of Anti-SARS-CoV-2 Antibodies in Persons With Mild Covid19. N Engl J Med (2020) 383(11):1085-7. doi: 10.1056/nejmc2025179

38. Long QX, Tang XJ, Shi QL, Li Q, Deng HJ, Yuan J, et al. Clinical and Immunological Assessment of Asymptomatic SARS-CoV-2 Infections. Nat Med (2020) 26(8):1200-4. doi: 10.1038/s41591-020-0965-6

39. Liu W, Fontanet A, Zhang PH, Zhan L, Xin ZT, Baril L, et al. Two-Year Prospective Study of the Humoral Immune Response of Patients With Severe Acute Respiratory Syndrome. J Infect Dis (2006) 193:792-5. doi: 10.1086/ 500469

40. Bourges D, Zhan Y, Brady JL, Braley H, Caminschi I, Prato S, et al. Targeting the Gut Vascular Endothelium Induces Gut Effector Cd8 T Cell Responses via Cross-Presentation by Dendritic Cells. J Immunol (2007) 179:5678-85. doi: 10.4049/jimmunol.179.9.5678 
41. Wakim LM, Smith J, Caminschi I, Lahoud MH, Villadangos JA. AntibodyTargeted Vaccination to Lung Dendritic Cells Generates Tissue-Resident Memory CD8 T Cells That Are Highly Protective Against Influenza Virus Infection. Mucosal Immunol (2015) 8:1060-71. doi: 10.1038/mi.2014.133

42. McKenzie BS, Corbett AJ, Johnson S, Brady JL, Pleasance J, Kramer DR, et al. Bypassing Luminal Barriers, Delivery to a Gut Addressin by Parenteral Targeting Elicits Local IgA Responses. Int Immunol (2004) 16:1613-22. doi: 10.1093/intimm/dxh163

43. Kathuria N, Kraynyak KA, Carnathan D, Betts M, Weiner DB, Kutzler MA. Generation of Antigen-Specific Immunity Following Systemic Immunization With DNA Vaccine Encoding CCL25 Chemokine Immunoadjuvant. Hum Vaccines Immunother (2012) 8:1607-19. doi: 10.4161/hv.22574
Conflict of Interest: The authors declare that the research was conducted in the absence of any commercial or financial relationships that could be construed as a potential conflict of interest.

Copyright (c) 2021 Jagtap, Bhor, Bhowmick, Kasarpalkar, Sagvekar, Kulkarni, Pathak, Chatterjee, Dolas, Palav, Kaginkar, Bhagat, Munshi, Parikh, Agrawal, Pawar, Kaneria, Mahale, Shastri and Patel. This is an open-access article distributed under the terms of the Creative Commons Attribution License (CC BY). The use, distribution or reproduction in other forums is permitted, provided the original author $(s)$ and the copyright owner(s) are credited and that the original publication in this journal is cited, in accordance with accepted academic practice. No use, distribution or reproduction is permitted which does not comply with these terms. 